\title{
Density based Traffic System and Collision Alert at Intersection of Roads
}

\section{Vaishali $B^{1 *}$ and Jeyapriya $A^{2}$}

${ }^{1}$ Department of Electrical Engineering, Universite du Quebec a Trois-Rivieres, Québec, Canada

${ }^{2}$ CSE, Sri Ramakrishna Engineering College, Coimbatore, Tamil Nadu, India

\begin{abstract}
Internet of things is the collection of gadgets and embedded objects to gather and exchange data by the usage of internet. The internet of things with intelligent transport system (ITS) has become the elucidation for the navigation of vehicles, traffic signal control systems, and automatic number plate identification. It has been evolved in applications for providing contemporaneous data and feedback from diverse sources such as weather information, parking guidance and also the gentrify traffic information from time to time. The proposed system focuses on dynamic traffic light sequence based on the traffic density in order to decrease the rate of accidents at intersection of roads. The obstacles that may or may not occur at intersection are recognized and warned by usage of an alert system and a notification is generated. The traffic management is used to supervise and retrieve current status of traffic and violators are discovered. This is mainly done to evacuate the traffic congestion and accidents at peak hours.
\end{abstract}

Keywords: Intelligent transport system; Red light; Runner; HMM; SVM; Hot spot

\section{Introduction}

In recent years, vehicle utilization has increased tremendously. Due to this, road traffic ambience has become more problematic and confusing. Accidents that occur at intersections of roads are due to some failure in traffic signal systems and drivers' knowledge. When the operations of traffic management systems are ameliorated with the help of security and proficiency of transportation systems, the general traffic system utilizes stable signalling times at intersections and does not provide any importance to emergency vehicles such as ambulances, fire-fighters and police cars thereby leading to a loss of lives, damage or destruction of property, and rise in fuel costs, pollution and congestion. The requirement of the sensor node is to manage traffic in a specific area by the usage of different devices that can measure and determine various physical traffic parameters like density and collision. A collision warning system operates as follows: a sensor installed at the front end of a vehicle constantly scans the road ahead for vehicles or obstacles. If such an obstacle is found, the system determines whether the vehicle is in imminent danger of crashing, and if so, a collision warning is given to the driver. The sensors used, fulfil the tasks of obstacle detection and tracking, which is the basis of collision warning sensing techniques (Figure 1).

Here we propose a system that generates the traffic light signals based on the density or the number of vehicles in the road which is a contradict to the old method. Where there are regular time intervals to all roads irrespective of their traffic voluminous. This type of traffic light signalling system is incorporated in almost of the metropolitans' cities. In this method to monitor and manage traffic, the density of traffic is

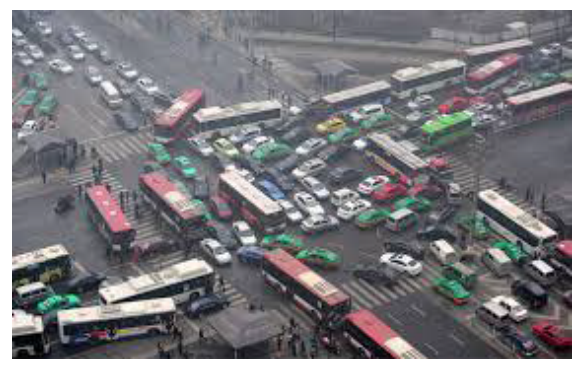

Figure 1: Collision at intersection of roads. measured using IR sensors; these sensors are placed on either sides of the road. The sensors output is given to a microcontroller then this data is processed in it. Thus, depending on density of traffic the timing of traffic lights are appropriately set. The ultrasonic sensors are also used to detect any collision or obstacles and if any, is detected warned by an alarm and warning message.

\section{Literature Survey}

The vehicle collision avoidance as well as the RLR [1] activity intersection is identified. When the RLR is detected the neighbouring vehicles are instructed to slow down. It uses on-scooter solution using smartphones. Using the GPS present in the smartphone behaviour of the scooter driver is known.

The red signal violators are predicted by infrastructure based and vehicle to roadside communication. The collision occurs basically because of last second turns and also because of the RLR violators. This [2] uses two schemes (1) for the enhancement of prediction rate (2) to find the status about the drivers.

The areas that high at risk are called as hot spot. The hot spots [3] are identified so the risk exposure would be minimized. The hot spot technique is not very effective for minor collisions.

The traffic exposure is considered as the incidents that occur on the roadway. The risk factor [4] for the occurrences of the incident is calculated. The calculation will help in the observation of traffic. This uses a hypotheses function. It's based four stages were each stage focus on simple, redundant and complex incidents

The collisions between the motor vehicles and pedestrians are more intense in regions where there is no traffic signal. It uses novel based

*Corresponding author: Vaishali B, Department of Electrical Engineering, Universite du Quebec a Trois-Rivieres, Québec, Canada, Tel: 015144737068; E-mail: vaishalibabuskct@gmail.com

Received May 13, 2017; Accepted June 26, 2017; Published June 28, 2017

Citation: Vaishali B, Jeyapriya A (2017) Density based Traffic System and Collision Alert at Intersection of Roads. J Electr Electron Syst 6: 229. doi: 10.4172/2332 0796.1000229

Copyright: @ 2017 Vaishali B, et al. This is an open-access article distributed under the terms of the Creative Commons Attribution License, which permits unrestricted use, distribution, and reproduction in any medium, provided the original author and source are credited. 
approach which uses the data about the characteristics of road and the surroundings of neighbourhood [5].

The double intersection roads have become cause to traffic congestion especially at rush hours. These kind of traffic that occur at double intersections include the roads are frequently used with not often used roads that have a wide median strip [6]. This type of intersections has a non-prior section in the middle to connect the prior road at the two sides, roads that are close to the banks of a river. The double-intersection should have a usual signalized intersection and for maximum capacity of vehicles there should be linked traffic signal control.

\section{Proposed System}

Traffic congestion is another problem that occurs now in most of the advanced cities around the world. Traffic congestion has induced many censorious problems and challenges in the most of the highly populated cities. The tour to different places within the city is becoming more problematic for the travellers because of traffic. Because of the clogging problems, people lose time, miss possibilities, and get frustrated. Due to traffic clogging there is a loss of yield from workers, trade possibilities are lost, delivery gets retarded, and thereby the costs are spiked. As result traffic is becoming one of important and major inconvenient problems in huge developed cities. Some of the most important traffic investigations are clogging and accidents which have causes an ample waste of time, property destruction and environs pollution. This research presents a novel intelligent traffic administration system, based on Internet of things, which is featured by low cost, high scalability, high compatibility, easy to upgrade, to replace standard traffic management system. The proposed system can improve road traffic immensely. The Internet of Things is based on the Internet, network wireless sensing and detection technologies to realize the intelligent recognition on the tagged traffic object, tracking, monitoring, managing and processed automatically. This paper includes the following list of components ARM7 microcontroller, IR sensor, ULTRASONIC sensor, LCD display, BUZZER.

\section{IR sensor}

An infrared sensor is an electronic appliance that discharges in order to sense some aspects of the environment. An IR sensor can estimate the heat of an object as well as detects the motion. This kind of sensors measures evaluate only the infrared radiation rather than emitting it, which is called as a passive IR sensor. The infrared spectrum generally radiates some kind of radiations with the objects it contains. Infrared sensor can detect these kinds of radiations which are not visible to our eyes. The emitter is generally an IR LED detector and the detector is generally an IR photodiode which is sensitive to IR light of the same wavelength as that emitted by the IR LED. The immensity of the IR light receives the changed proportion of photodiode, resistance and the output voltages when the IR light falls on them (Figure 2).

\section{Ultrasonic sensor}

The alternate transmission and reception of sound waves are allowed by the ultrasonic propinquity sensors that are usually used in the special sound transducers. The transducer discharges the sonic waves and are reflected by an object and received back in the transducer. The ultrasonic sensor switches to the receive mode after the emission of the sound waves, the measure of the distance of the object from the sensor is proportional to the time advanced between the release and receive of sound waves (Figure 3 ).

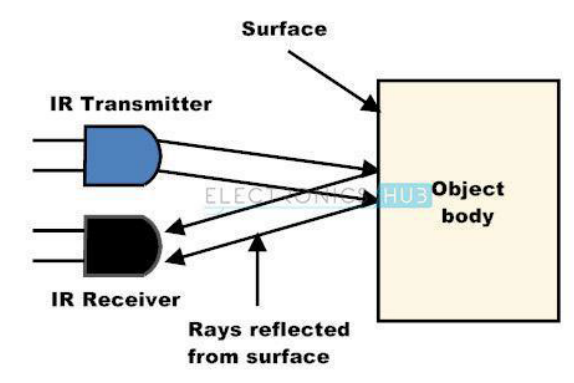

Figure 2: IR sensor.

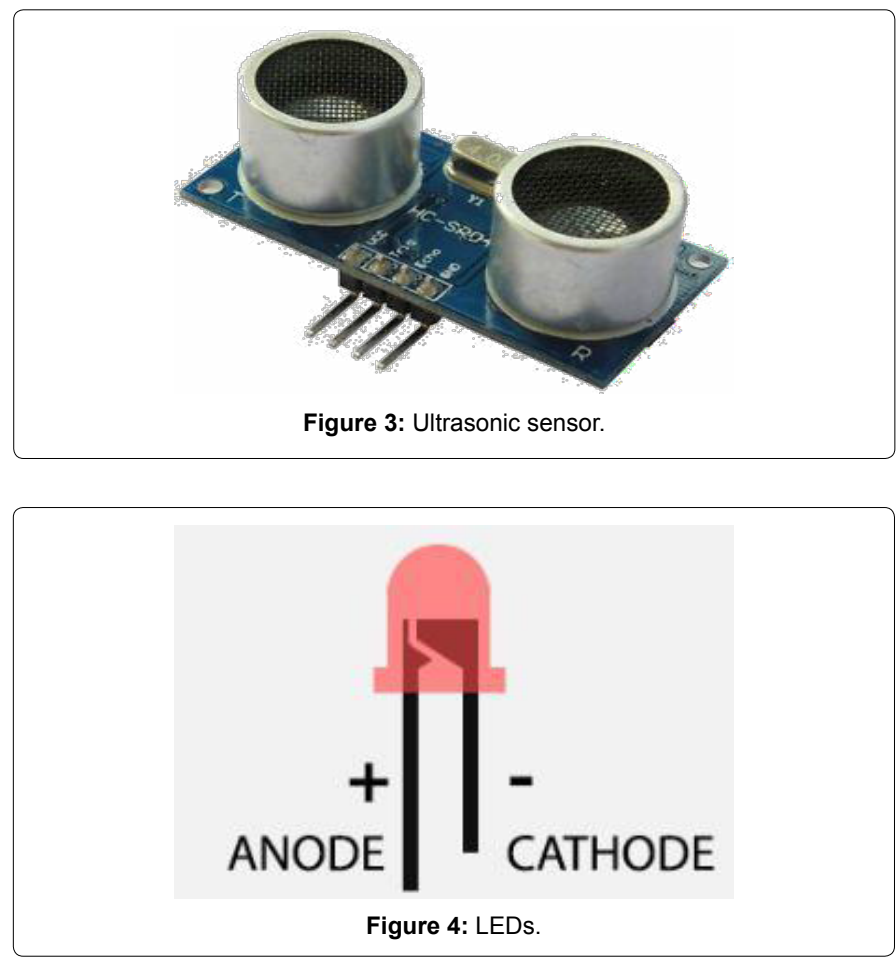

LED

LEDs are specifically designed so they make light of a certain wavelength and they're built into rounded plastic bulbs to make this light brighter and more focused. LEDs of red color generate light with a wavelength of about 630-660 nanometers-which happens to look red when we see it, while blue LEDs generate light with shorter wavelengths of about 430-500 nanometers, which we see as blue. The proficiency of simple tungsten lamp is almost 50 times lesser than an LED. When compared with 100 milliseconds for a tungsten lamp, the response time of the LED are 0.1 milliseconds faster (Figure 4).

\section{LCD display}

LCD is a technology used for small computers. It displays about 224 alphanumeric characters. The 8 data pins in the LCD are fetched to the ASCII 8-bit code of the character to LCD. However, the data can be converted to a character type array and it's sent one by one to the LCD. Data can be sent by the usage of LCD either in 8-bit or 4-bit mode. Two nibbles of data (First high four bits and then low four bits) are to be sent to complete a full eight-bit transfer in case of 4 -bit mode. The 8 -bit mode is preferred for use when the speed is required in an application 4-bit mode requires a minimum of seven bits (Figure 5). 
Citation: Vaishali B, Jeyapriya A (2017) Density based Traffic System and Collision Alert at Intersection of Roads. J Electr Electron Syst 6: 229. doi: 10.4172/2332-0796.1000229

Page 3 of 4

\section{Buzzer}

Buzzers are often employed to grant the user or operator an audio indication of the phase of a mechanical device. For instance, buzzing someone in || refers to opening an electromechanical door lock, which generates a buzz, stating that the lock has been moved to the open position. Buzzers are mainly being utilized on computers, generally to stipulate error conditions. These buzzers may go off if there is a fault present in the component of the device. Buzzers are also utilized for alarm systems (Figure 6).

\section{ARM Microcontroller}

It's an enhanced RISC machine in the RISC framework. There are small in size, less hardware cost, power consumption is minimum. The memory operation is performed by the general purpose register. Most commonly used in all mobile phones (Figure 7).

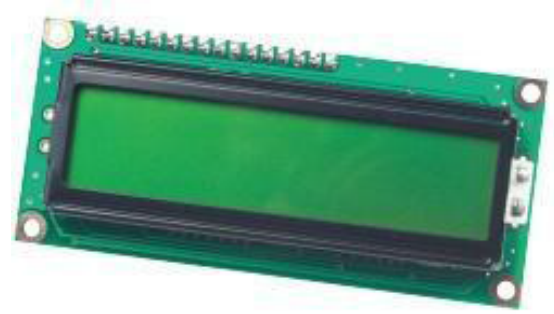

Figure 5: LCD display.

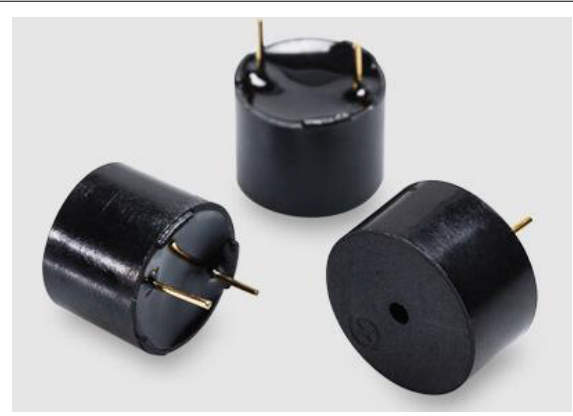

Figure 6: Buzzers

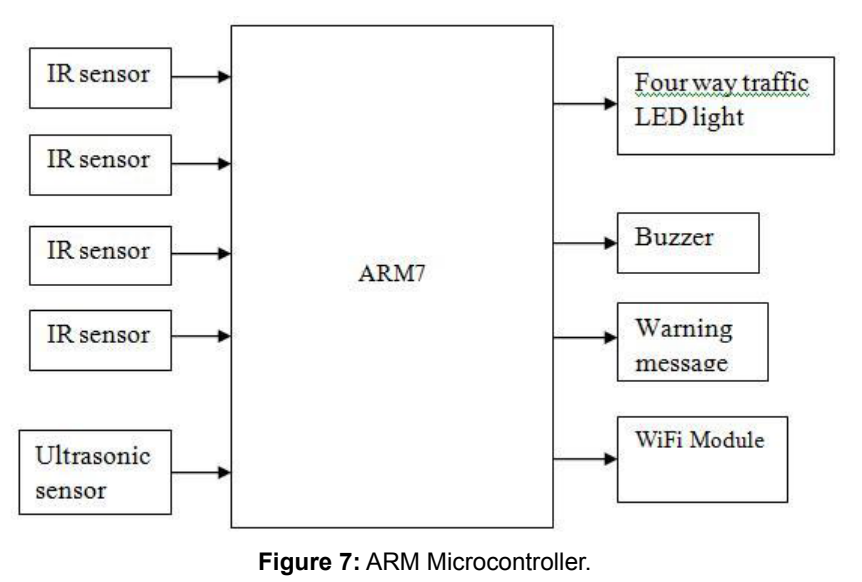

\section{Implementation Phase}

The implementation phase of collision avoidance at intersection of roads consists of 3 modules: dynamic traffic light sequence, obstacle detection and monitoring traffic.

Figure 8 represents the architecture of the proposed system. The Infrared (IR) sensor are used for count the number of vehicles i.e., to determine the maximum capacity for dynamic control of signals. The ultrasonic sensors are used to detect the obstacles that may occur at intersections. The ultrasonic sensors are used since it detects the obstacles based on the sound so that the false alarm or false negative can be reduced. The warning message is displayed in a LCD display and alarm is given using a buzzer. The traffic management with internet of things is done to identify the violators, monitor the current state of traffic and to reduce the accidents at intersection.

The components that are used in the architecture are explained in Section III. The overall prototype of the system is depicted in Figure 9.

\section{Dynamic traffic light sequence}

In this module, the traffic signal at each side of the road is of $20 \mathrm{sec}$. The density or voluminous of each road is the total number of vehicles is calculated. The count of the total number of vehicles is measured using the IR sensor. Based on the highest count of the vehicles at each side of the road the traffic signal is turned green. This is done mainly minimize traffic clogging at rush time hours. All the traffic signal LEDs are connected to the GPIO pins of ARM controller. At normal traffic signal works on 2 modes are Time-basis mode and Density based mode. In time basis mode the traffic signals changed based on some time interval. This time interval is determined in the coding part of the

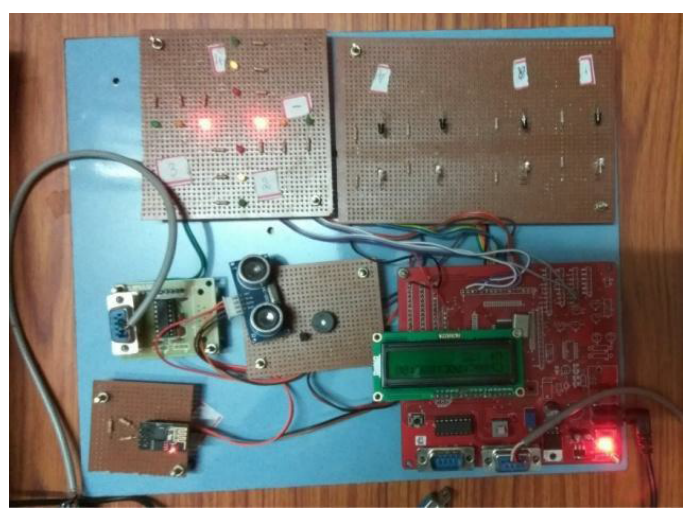

Figure 8: Architecture of the proposed system.

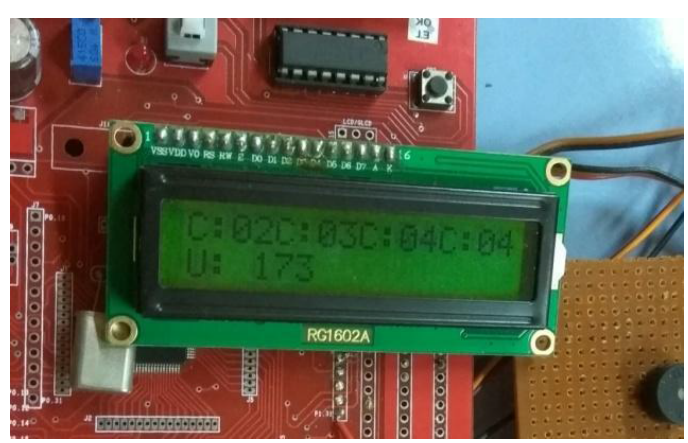

Figure 9: Prototype of the collision avoidance system. 
Citation: Vaishali B, Jeyapriya A (2017) Density based Traffic System and Collision Alert at Intersection of Roads. J Electr Electron Syst 6: 229. doi: 10.4172/2332-0796.1000229

arm controller. This mode will active only when the all ways of traffic will be normal condition and weather they have equal traffic in all the ways. In density basis mode the traffic signals changed based on density of traffic present in each way. This mode will only activate when the traffic will be exited the normal condition (Figure 10).

\section{Obstacle detection}

In this module, the ultrasonic sensor is used to detect whether there is traffic jam or collision between vehicles or any obstacle has occurred suddenly is identified. The obstacles are identified based upon their sound. If an obstacle is detected an alarm is generated from the alert system. The alert system includes alarm and LCD display. The detection of ultrasonic sensor is alerted using a alarm and also an warning message is sent (Figure 11).

\section{Monitoring traffic}

The traffic information includes about whether there is any collision occurred or any obstacle detected. It also includes the information about the traffic whether it's high or low. The traffic information can be viewed in the browser. This information is updated using wifi module with UART part (Figure 12).

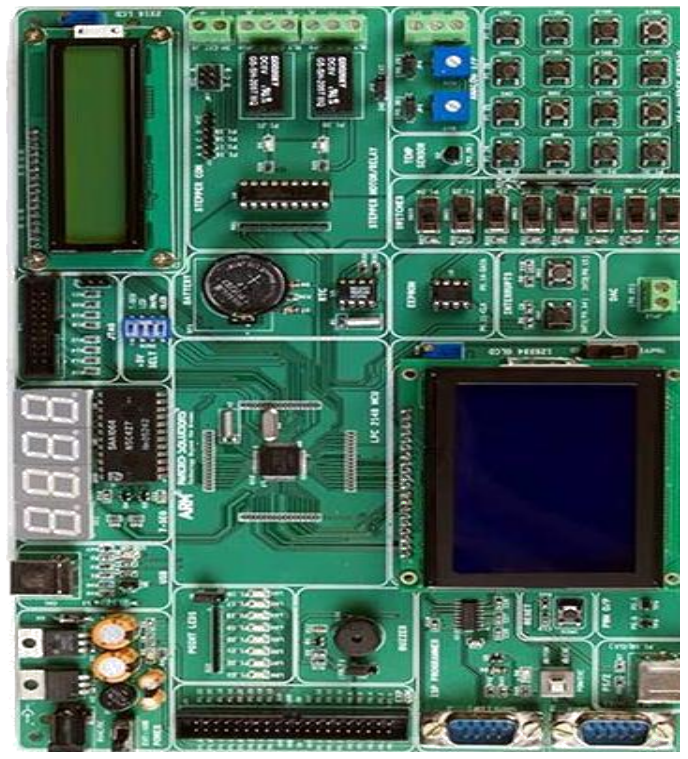

Figure 10: Density of traffic.

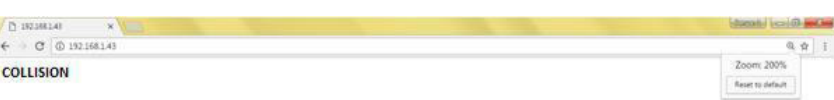
COLISION

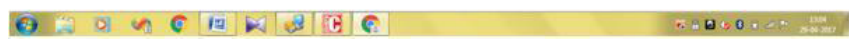

Figure 11: Obstacle is detected.

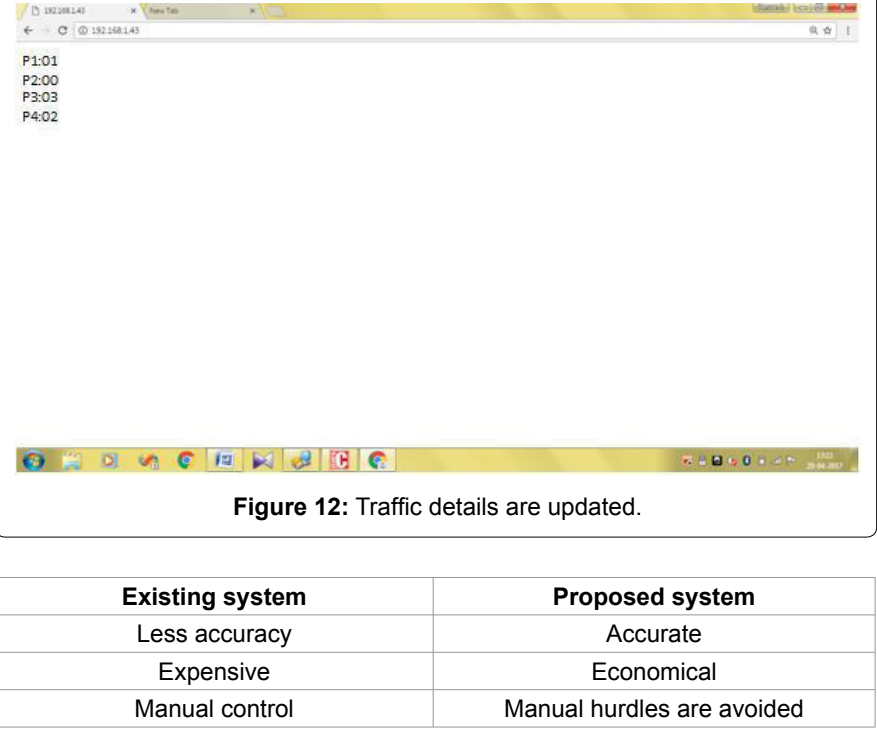

Table 1: Comparison of existing and proposed system.

\section{Results}

In order to minimize the number of accidents those exist at the intersection of road for the welfare of vehicle drivers, pedestrians. This is not only caused by the collision of vehicles but also by the disobeyers of the traffic rules. The traffic clogging is depleted by the usage of IR sensor. This calculates the voluminous of vehicles at each side of the road and the traffic light sequence is changed accordingly. To ensure the safety of the vehicle drivers an ultrasonic sensor is used. If any obstacle is detected an alarm is generated thereby alerting the neighbouring vehicles. The identified obstacles and traffic information are updated to the webpage through $\mathrm{Wi}$-Fi module. The human intervention is avoided to minimize accidents (Table 1).

\section{Conclusion}

The traffic system is used for decreasing the rate of accidents at intersection and the traffic is monitored. The traffic congestion and maximum capacity of vehicles are depleted by designing the signalized roads at intersections of roads. The identification of the critical regions is to be monitored in order to decrease the rate of the occurrence of accidents.

\section{References}

1. Huang KS, Chiu PJ, Tsai HM, Kuo CC, Lee HY, et al. (2016) RedEye: Preventing collisions caused by Red-light-Running scooters with smartphones. IEEE Transaction on Intelligent Transportation System 17: 1243-1257.

2. Wang L, Zhang L, Zhang WB, Zhou K (2009) Red Light Running Prediction for dynamic All-red Extension at signalized Intersection. Proceedings of the 12th International IEEE conference on Intelligent Transportation system.

3. Washington S, Haque M, Oh J, Lee D (2014) Applying Quntile regression for modelling equivalent property damage only crashes to identify accident blacspots. Accident Analysis and Prevention 66: 136-146.

4. Elvik R (2015) Some implications of an event-based definition of exposure to the risk of road accident. Accident Analysis and Prevention 76: 15-24.

5. Quistberg DA, Howard EJ, Ebel BE, Moudon AV, Saelens BE, et al. (2015) Multilevel models for evaluating the risk of pedestrian-motor vehiclecollisions at intersections and mid-blocks. Accident Analysis and Prevention 84: 99-111.

6. Vu MT, Nguyen VP, Nguyen VB, Nguyen TA (2016) Methods for deigning Signalized double-intersection with mixed Traffic in Vietnam. Procedia engineering, pp: 131-138. 